\title{
Evaluation of three-dimensional arterial spin labeling perfusion imaging for the pathological investigation of musculoskeletal tumors
}

\author{
LINGBIN XU ${ }^{1}$, LEIMING $\mathrm{XU}^{2}$ and HAIDONG $\mathrm{ZHU}^{1}$ \\ ${ }^{1}$ Department of Radiology, Ningbo Medical Center Lihuili Eastern Hospital Taipei Medical University \\ Ningbo Medical Center, Ningbo, Zhejiang 315040; ${ }^{2}$ Department of Radiology, The Second Affiliated \\ Hospital Zhejiang University School of Medicine, Hangzhou, Zhejiang 310000, P.R. China
}

Received August 13, 2017; Accepted March 7, 2018

DOI: $10.3892 /$ etm.2018.6030

\begin{abstract}
The present study aimed to assess the clinical utility of three-dimensional arterial spin labeling (3D-ASL) perfusion imaging in discriminating between benign, intermediate and malignant musculoskeletal tumors, as well as to analyze the correlation between tumor blood flow (TBF) and microvessel density (MVD). 3D-ASL was performed on 44 patients with musculoskeletal tumor using a 3.0-T magnetic resonance system to obtain TBF values prior to surgery. TBF was independently measured by two radiologists. The TBF values of different groups were compared by one-way analysis of variance. A receiver operating characteristic (ROC) curve was applied to assess the threshold and diagnostic reliability of TBF. Immunohistochemical staining of tumor specimens was performed using a cluster of differentiation 34 monoclonal antibody to calculate MVD counts. The correlation between TBF and MVD counts was analyzed using correlative analysis. Pathology results for a total of 44 cases were obtained by surgery. Good interobserver agreement was found for the TBF values independently determined by the two radiologists (intra-class correlation coefficient test, $0.891 ; \mathrm{P}<0.05$ ). TBF and MVD values in the malignant group were significantly higher compared with that of the benign, and intermediate groups. No significant difference was found between the TBF and MVD values of the benign, and intermediate groups. According to the ROC analysis, the area under the curve was largest (0.951) when
\end{abstract}

Correspondence to: Dr Haidong Zhu, Department of Radiology, Ningbo Medical Center Lihuili Eastern Hospital Taipei Medical University Ningbo Medical Center, 1111 Jiangnan Road, Ningbo, Zhejiang 315040, P.R. China

E-mail: haidongzhu11@163.com

Abbreviations: 3D-ASL, three-dimensional arterial spin labeling; TBF, tumor blood flow; MVD, microvessel density; ROI, regions of interest; SAR, absorption rate

Key words: musculoskeletal tumors, arterial spin labeling, pathology, comparative study, microvessel density
$45.5 \mathrm{ml} / \mathrm{min} / 100 \mathrm{~g}$ was considered as the TBF cut-off value in the diagnosis. The diagnostic sensitivity and specificity were 90.5 and $100 \%$, respectively. Additionally, a significant positive correlation was found between TBF and MVD (r, $0.784 ; \mathrm{P}<0.05)$. The results of the present study suggest that $3 \mathrm{D}$-ASL is valuable in discriminating between benign, intermediate and malignant musculoskeletal tumors. 3D-ASL may be utilized to evaluate angiogenesis in musculoskeletal tumors in vivo.

\section{Introduction}

Musculoskeletal tumors are frequent recording an incidence of $3 / 1,000$ in USA, but only $0.67 \%$ are malignant, that pose a serious public health problem in the modern world (1). Malignant musculoskeletal tumors are associated with high mortality and disability rates (2-4). Although it is not possible to determine the exact cost of the burden of malignant musculoskeletal tumors, a good example comes from a model for the productivity costs of cancer mortality that projected the annual expenses for USA at approximately $\$ 115.8$ billion in the year 2000 , and $\$ 147.6$ billion for 2020 (5). It is very important to raise global awareness of the growing burden of malignant musculoskeletal tumor. Benign and malignant musculoskeletal tumours are notoriously difficult to differentiate and represent a clinical, radiological and histological challenge. Meanwhile, accurate prediction of the grade of tumor cell before surgical removal could affect the type of surgery and need for adjuvant therapy, but it is not always possible with the current imaging methods. Typical imaging characteristics exist for common benign lesions such as lipomas and hemangiomas. In contrast, no specific imaging features and clinical manifestations exist for certain musculoskeletal tumors. Therefore, distinguishing between malignant tumors and benign tumors is difficult using common methods $(6,7)$. At present, pathological inspection is clearly the 'gold standard' for identifying pathological changes, and histopathology is the most objective method because it involves the direct examination of pathological changes. However, the inherent shortcoming of histopathology is that it involves invasive inspection. Therefore, there is an urgent 
need for clinicians to identify non-invasive approaches that are comparable to biopsies to qualitatively describe lesions.

Functional magnetic resonance imaging has become commonly used worldwide and has great potential for characterizing pathological changes $(8,9)$. Functional magnetic resonance imaging includes perfusion-weighted imaging, diffusion-weighted imaging and magnetic resonance spectroscopy.

Tumor angiogenesis is the formation of new blood vessels by budding or sprouting from the existing vasculature (10). Angiogenesis plays a key role in the tumorigenesis, invasion and metastasis of solid tumors $(11,12)$ and is considered a sign of tumor invasion because the resulting rich vascular network provides tumor cells with sufficient oxygen and nutrients and contributes to tumor metastasis (13). The microvessel density (MVD) count is the standard method used to analyze tumor angiogenesis (14). Dynamic observations of angiogenesis and the functional status in living organisms cannot be performed due to the invasiveness and in vitro of such procedures.

Three-dimensional arterial spin labeling (3D-ASL) can perform using a pseudo-continuous arterial spin labeling sequence that uses blood as an endogenous contrast agent, allowing noninvasive perfusion measurements to be performed without gadolinium administration. The objective of our study is to evaluate 3D-ASL perfusion imaging in discriminating between benign, intermediate and malignant musculoskeletal tumors as well as to analyze the correlation between tumor blood flow (TBF) and MVD.

\section{Patients and methods}

Patients and MR imaging. 3D-ASL was performed on 44 patients (20 males and 24 females with a median age of 27 years and an age range of 9 to 57 years) from March 2013 to January 2014. The patients were selected according to the following inclusion criteria: i) The presence of low extremity or pelvic musculoskeletal tumors; ii) surgical resection performed and a definite pathologic diagnosis obtained within two weeks; and exclusion criteria: i) the general condition cannot tolerate MR examination; ii) undergo radiotherapy or chemotherapy treatment prior to 3D-ASL; and iii) the presence of vascular stenosis in extremity or pelvis.

The patients were divided into benign, intermediate and malignant groups according to the International Classification of Diseases for Oncology (15).

All subjects were scanned on a 3.0-T magnetic resonance (MR) system (Discovery 750; GE Healthcare, Milwaukee, WI, USA) with use of a 32-element phased-array abdominal coil (GE Healthcare), an ADW4.6 workstation and Functool software. The examination protocol comprised axial T1-weighted image with fat-suppressed, T2-weighted images with fat saturation and 3D-ASL. The T1-weighted, T2-weighted scan parameters were as follows: repetition time (TR) 250/4,000 $\mathrm{msec}$; echo time (TE) 25/76 msec; slice thickness/gap 4/0 mm; number of excitations (NEX) 2.0; matrix 320x256; and field of view (FOV) 30x30-40x40 cm (selected to accommodate the size of the tumor). For 3D-ASL, calibration was performed before scanning using the same slice thickness, gap, matrix and FOV as T2-WI and with TE $10.4 \mathrm{msec}$ and TR 4,552 $\mathrm{msec}$, post label delaey (PLD) 1,525 msec, NEX=2.0.
This study was approved by the Committee for the Protection of Human Subjects of the Institutional Review Board of Ningbo Medical Treatment Center Lihuili Hospital, and informed consent was obtained from all subjects.

Image processing and data analysis. After exporting the raw ASL data, post-processing was performed to obtain the TBFmaps using the GE ADW4.6 workstation, followed by slice-by-slice registration and fusion with the T2-WI images by radiologists. The brightly colored regions in TBF images represent areas of high perfusion. Regions of interest (ROIs) for quantitative analysis were manually placed on the highest perfusion slice at TBF maps independently by two radiologists (more than 15 years of experience) who were dedicated to musculoskeletal tumor study in our hospital. Necrosis, cystic areas, hemorrhage and calcification were excluded by T1-weighted, T2-weighted. In principle, the area of the ROI should be $20-35 \mathrm{~mm}^{2}$. Three ROIs were measured, and the mean value was calculated.

Pathologic examination. CD34 (cat. no. MAB-0034 QBEnd/10) and DAB kit (cat. no. Kit-0014; MaxVision Biosciences Inc., Fuzhou, China) was used for immunohistochemical staining of tumor specimens. CD34 monoclonal antibody was used to label vessel endothelial cell. The CD34 monoclonal antibody and the DBA kit were both purchased from Maxin Biotech Co., Ltd. (Fuzhou, China). The staining was conducted according to manufacturer's instruction. MVD counts were performed according to Weidner's method (14). We found neovascular 'hotspots' by scanning the tumor sections at low power (x40). Individual microvessel counts were then performed on a $x 400$ field within the neovascular hotspot. Any individual endothelial cell or endothelial cell cluster that was positive for CD34 (observed as brown staining) and was clearly separate from an adjacent cluster was considered to be a single, countable microvessel. Microvessels larger than 8 erythrocyte diameters were not considered in the vessel counts. Any counts were performed by two investigators using a double-headed light microscope simultaneously, both had to agree on what constituted a single microvessel before any vessel was included in the count. Each count was expressed as the highest number of microvessels identified within any x400 field.

Statistical analysis. All statistical analyses were performed using the SPSS 21.0 package for Windows (SPSS, Inc., Chicago, IL, USA). $\mathrm{P}<0.05$ was considered to indicate a statistically significant difference. An intra-class correlation coefficient test was used to determine if there was good inter-observer agreement in the TBF values measured independently by two experienced radiologists. Group differences in TBF were assessed using one-way analysis of variance followed by the Least Significant Difference test to compare TBF among three groups. A receiver operating characteristic (ROC) curve was computed to display the sensitivity and specificity of TBF in predicting the nature of musculoskeletal tumors. The area under the receiver operating characteristic curve (AUC) of TBF was calculated, and the cut-off value was then calculated to achieve the maximum accuracy percentage. Correlations between TBF and MVD were assessed by Pearson's correlation analysis. 
Table I. Histopathological description of tumor and subtypes of 44 cases included in the present study.

\begin{tabular}{lc}
\hline Subtype & No. of patients \\
\hline Benign & \\
Chondroblastoma & 2 \\
Hemangioma & 1 \\
Osteochondroma & 1 \\
Enchondroma & 3 \\
Fibroma & 2 \\
Aneurismal bone cyst & 2 \\
Intermediate & \\
Desmoid type fibromatosis & 2 \\
Giant cell tumor of bone & 5 \\
Solitary fibrous tumor & 2 \\
Malignant & \\
Osteosarcoma & 11 \\
Metastases involving bone & 3 \\
Liposarcoma & 1 \\
Primitive neuroectodermal tumor & 1 \\
Plasma cell myeloma & 1 \\
Pleomorphic undifferentiated sarcoma & 1 \\
Epithelioid hemangioendothlioma & 1 \\
Ewing's sarcoma & 1 \\
Sclerosing epithelioid fibrosarcoma & \\
\hline
\end{tabular}

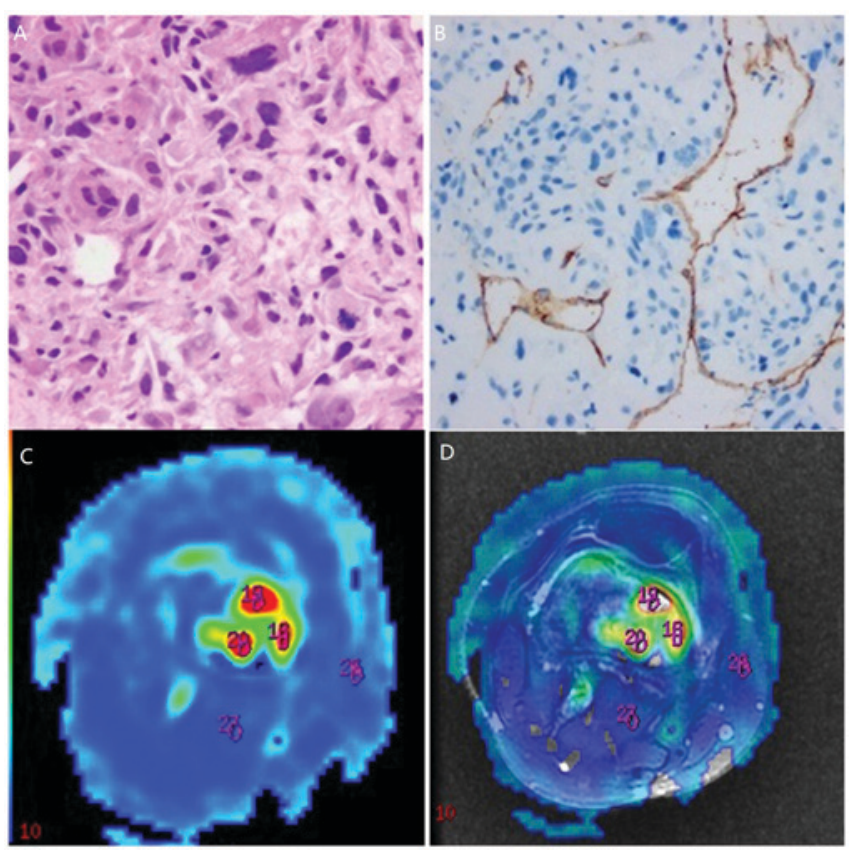

Figure 1. A 16-year-old female patient with osteosarcoma in the malignant group. (A) Routine pathological (H\&E, x400) images, (B) Immunohistochemical CD34 staining (H\&E, $\mathrm{x} 400)$. The vessel in malignant tumor are present of heterogeneity. There are large differences in the diameter and morphology of microvessels in malignant tumors. Some of them only contain individual endothelial cell or endothelial cell cluster which was positive for $\mathrm{CD} 34$ are varies in diameter and morphology. (C and D) TBF images and combined TBF and routine T2-WI images show the malignant tumor has heterogeneous higher perfusion. The solid-appearing mass has bright color than that of normal muscle in the same slice. $\mathrm{H} \& \mathrm{E}$, hematoxylin and eosin; TBF, tumor blood flow.

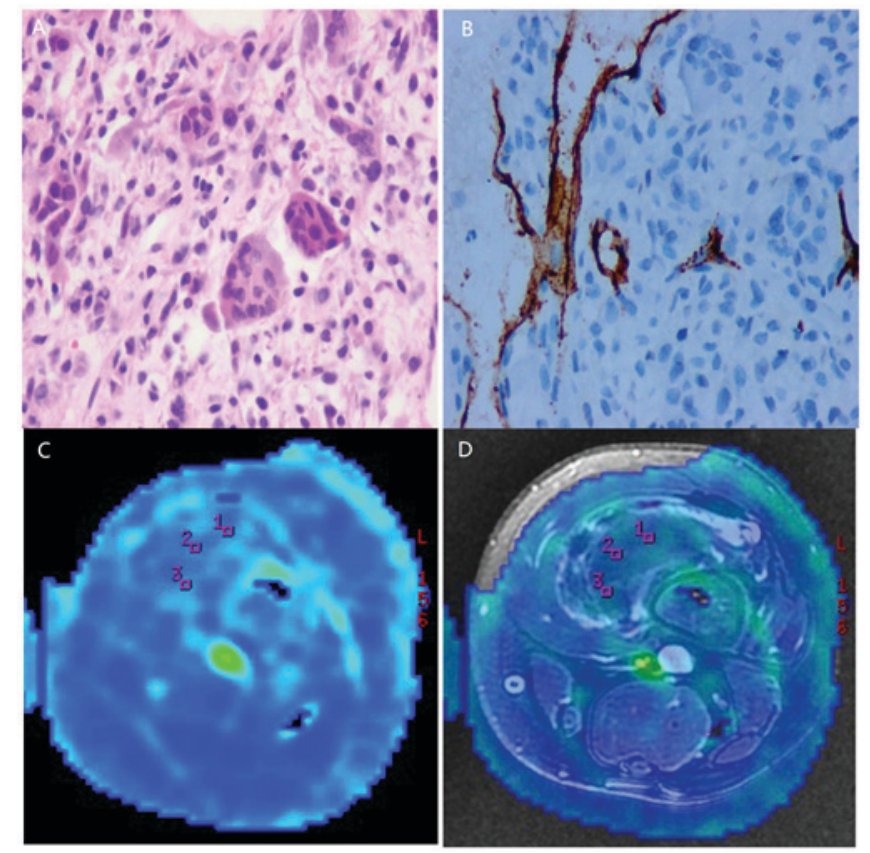

Figure 2. A 24-year-old male patient with a giant cell tumor of the bone in the intermediate group. (A) Routine pathological (H\&E, x400) images. (B) Immunohistochemical CD34 staining (H\&E, x400). Single endothelial cell or cluster positive for $\mathrm{CD} 34$ and stained brown are rare. (C and D) TBF images and combined TBF and routine T2-WI images indicate that the MVD value of the intermediate group is significantly lower than the MVD values of the malignant groups. H\&E, hematoxylin and eosin; TBF, tumor blood flow; MVD, microvessel density.

\section{Results}

There were 12 subjects in the benign group, 10 in the intermediate group and 22 in the malignant group. Detailed diagnoses are reported in Table I. Routine pathological sections were obtained in all subjects (Figs. 1A, 2A and 3A). All tumor sections were positive for CD34 (Figs. 1B, 2B and 3B). The MVD counts were $10.38 \pm 4.58$ and $14.64 \pm 6.69$ in the benign and intermediate groups, respectively, and $32.97 \pm 11.61$ in the malignant group.

Good inter-observer agreement was found for the TBF measurements made by the two observers as assessed by the intra-class correlation coefficient test $(\mathrm{ICC}=0.891$, $\mathrm{P}<0.05$ ). The brightly colored regions in TBF images obtained by 3D-ASL represent areas of high perfusion. The solid-appearing parts of malignant tumors were brighter in color than the benign and intermediate tumors (Figs. 1C, 2C and 3C), which can be observed more obviously in fusion images (Figs. 1D, 2D and 3D). Additionally, necrotic or cystic areas demonstrated perfusion defects. Significant differences were found in the MVD and TBF among the three groups $(\mathrm{F}=28.33, \mathrm{P}<0.05, \mathrm{~F}=32.34, \mathrm{P}<0.05$, respectively). The MVD and TBF results are displayed in Table II. The MVD and TBF values were higher for malignant tumors than for benign and intermediate tumors $(\mathrm{P}<0.05)$, and TBF was not significantly different between benign and intermediate tumors.

The ROC curve of malignant musculoskeletal tumors plotted in Fig. 4 demonstrates that the TBF $\mathrm{AUC}_{\mathrm{ROC}}$ is 0.951 and that the ROC cut-off value of TBF is $45 \mathrm{ml} \cdot \mathrm{min}^{-1} 100 \mathrm{~g} \mathrm{~g}^{-1}$ 
Table II. MVD and TBF of the musculoskeletal tumors of the three groups.

\begin{tabular}{lccc}
\hline Group & No. & $\begin{array}{c}\text { MVD } \\
(\text { Pieces/HPF })\end{array}$ & $\begin{array}{c}\text { TBF } \\
\left(\mathrm{ml} \cdot \mathrm{min}^{-1} \cdot 100 \mathrm{~g}^{-1}\right)\end{array}$ \\
\hline Benign & 12 & $10 \pm 4^{\mathrm{a}}$ & $30 \pm 10^{\mathrm{a}}$ \\
Intermediate & 10 & $15 \pm 6^{\mathrm{b}}$ & $30 \pm 12^{\mathrm{b}}$ \\
Malignant & 22 & $33 \pm 11$ & $84 \pm 29$ \\
F-value & & 28.33 & 32.34 \\
P-value & & $<0.05$ & $<0.05$ \\
\hline
\end{tabular}

aBenign group have lower MVD and TBF values than malignant group. 'Intermediate group have lower MVD and TBF values than malignant group. MVD, microvessel density; TBF, tumor blood flow; $\mathrm{HPF}$, high power field.

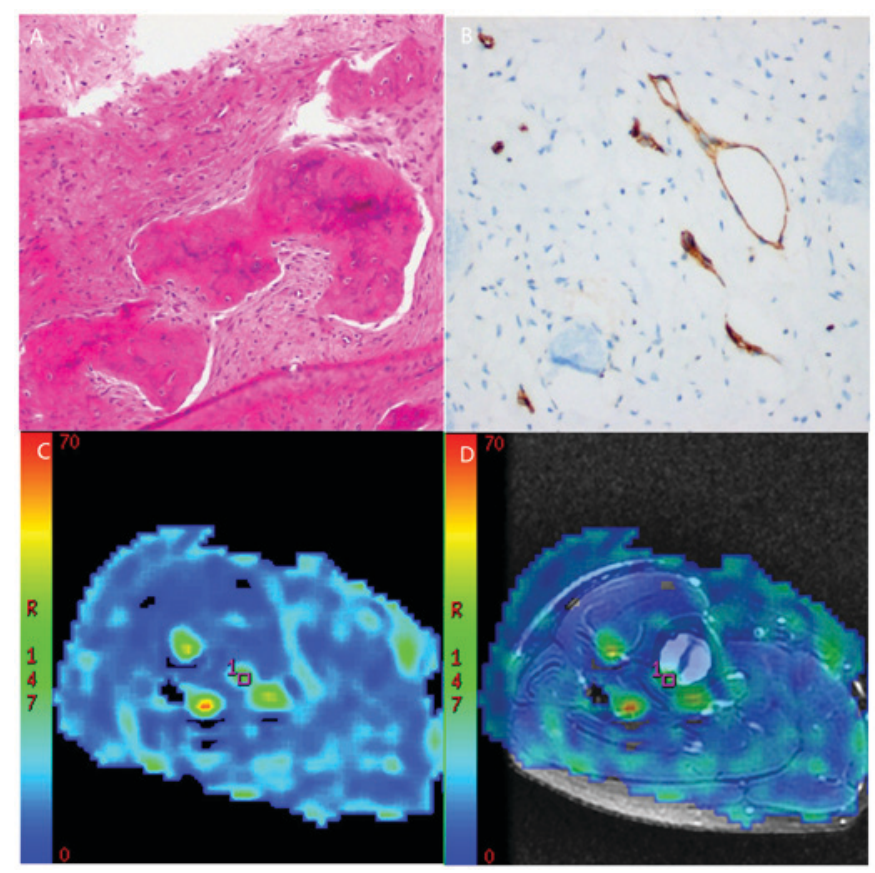

Figure 3. A 26-year-old female patient with fibrous dysplasia in the benign group. (A) Routine pathological (H\&E, x400) images. (B) Immunohistochemical CD34 staining (H\&E, x400). The figures indicate that the MVD value of the benign group is significantly lower than the MVD values of the malignant groups. (C and D) TBF images and combined TBF and routine T2-WI images. The bright regions represent areas of high perfusion. The benign tumors have lower perfusion. H\&E, hematoxylin and eosin; TBF, tumor blood flow; MVD, microvessel density.

$(\mathrm{P}<0.05)$. The diagnostic sensitivity and specificity are $90.5 \%$ and $100 \%$, respectively.

According to Pearson's correlation analysis, a significant positive correlation was found between TBF and MVD $(\mathrm{r}=0.784, \mathrm{P}<0.05)$, as demonstrated by the approximately linear positive correlation shown in Fig 5.

\section{Discussion}

Angiogenesis is a dynamic process that is related to many factors. Under physiological conditions, angiogenesis is

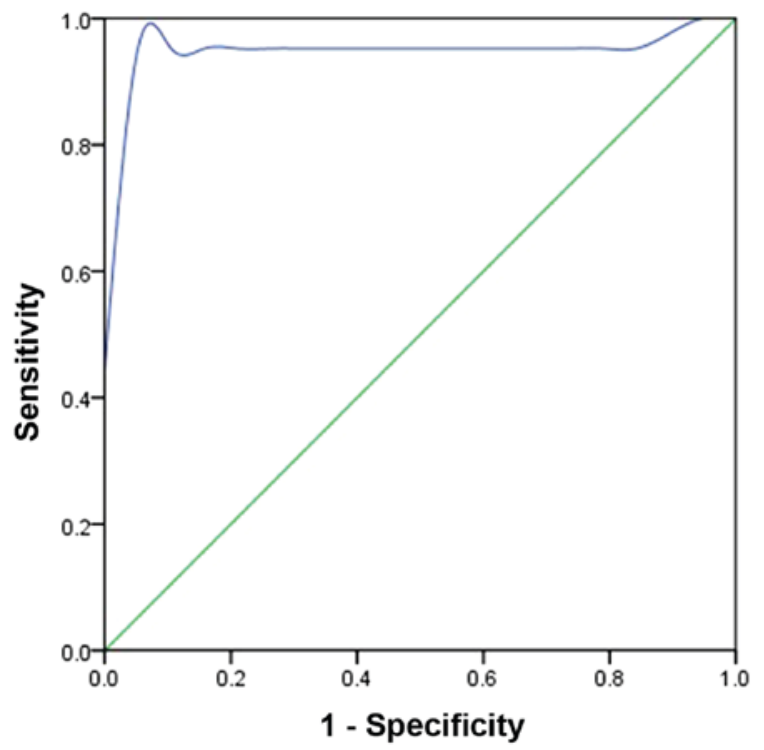

Figure 4. ROC curve of TBF shows good efficiency for diagnosing malignant tumors. ROC, receiver operating characteristic; TBF, tumor blood flow.

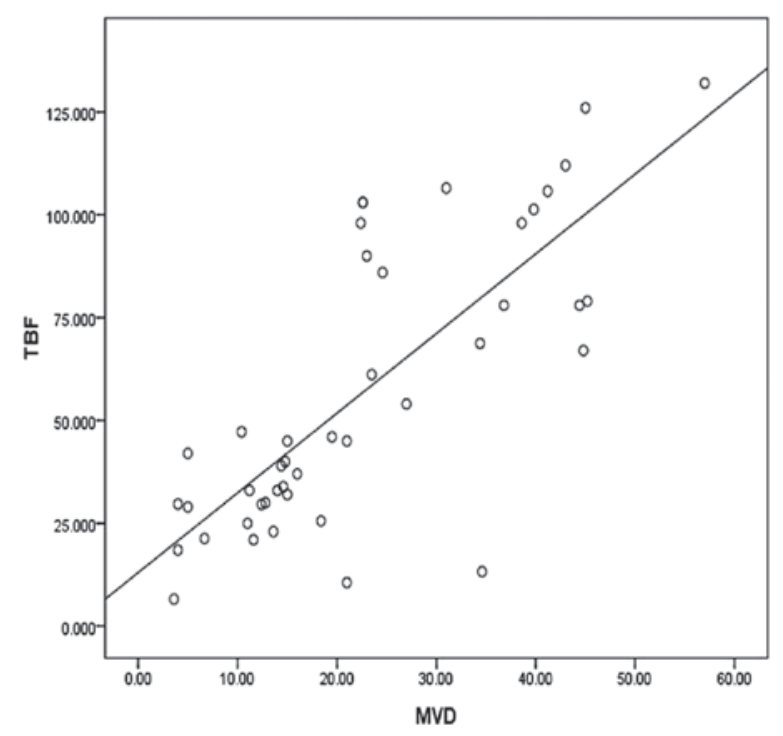

Figure 5. Correlation between TBF and MVD with a scatter plot and regression line. Two methods reached significant positive correlation $(r=0.784$, $\mathrm{P}<0.05)$. TBF, tumor blood flow; MVD, microvessel density.

strictly controlled by various factors to maintain the delicate balance between angiogenic and anti-angiogenic factors (16). Under pathological conditions, in which this regulation is out of balance, angiogenic factors surpass anti-angiogenic factors, and control is lost (17). The presence of many immature new blood vessels is an important feature of malignant tumors. Angiogenesis in tumors is closely related to the tumor growth rate, the grade of malignancy, the possibility of invasion or metastasis and the prognosis (18-20). Quantitatively analyzing angiogenesis is therefore critical. Since Weidner et al (14) first used MVD to evaluate tumor angiogenesis, many scholars $(21,22)$ have investigated and analyzed tumor angiogenesis in a variety of solid tumors using the MVD. At present, the MVD is the 'gold standard' for assessing tumor angiogenesis because it is an objective and sensitive measure. In the 
present study, differences in the MVD between the benign and malignant groups and between the intermediate and malignant groups were statistically significant $(\mathrm{P}<0.05)$. Thus, the MVD may reflect the nature and the degree of differentiation of musculoskeletal tumors to a certain extent. Luczynska and colleagues reached a similar conclusion in their study of prostate tumors (23). In our study, no significant difference in the MVD was found between the benign and intermediate groups. There are a couple possible explanations for this result. First, although we investigated various musculoskeletal tumor types, the sampling capacity of the study was limited. Second, tumor angiogenesis is not only related to the number of capillaries but also to the function of capillaries, which includes vascular endothelial cell and basement membrane integrity, blood vessel permeability and capillary flow. Therefore, further research on the subject is warranted. Although we agree that the MVD is the 'gold standard' for identifying angiogenesis in tumors, MVD counts cannot be extensively performed due to invasiveness as well as to in vitro assay defects (24).

Functional imaging can evaluate tumor angiogenesis based on the level of perfusion. CT perfusion imaging (25) and MR perfusion-weighted imaging (26) can both measure perfusion, but both modalities require the intravenous injection of contrast medium, which may be associated with contrast medium allergies or side effects to the viscera. CT examination is also associated with a risk of radiation exposure. Loizides et al (27) showed by Contrast Enhanced Ultrasound that the enhancement curves of benign and malignant tumors are different. This difference may contribute to the ability to distinguish between benign and malignant tumors of a similar size and location. However, the results were based on the analysis of semi-quantitative data; therefore, the reliability was not very good.

In this study, 3D-ASL was performed using a pseudo-continuous arterial spin labeling sequence that uses blood as an endogenous contrast agent, allowing noninvasive perfusion measurements to be performed without gadolinium administration. Inflowing blood is selectively labeled with the opposite magnetization of the destination tissue. The difference between a labeled image (tag) and an unlabeled image (control) can be used to calculate tissue perfusion. 3D-ASL uses a spin echo sequence as an acquisition approach to obtain a wide range of perfusion with a low specific absorption rate (SAR) and a high signal-to-noise ratio and tag rate. 3D-ASL, first applied clinically to examine the brain, has recently demonstrated some clinical value in body examinations (28). To date, ASL perfusion weighted-imaging has rarely been performed on musculoskeletal tumors in humans. However, a few studies using a rabbit VX2 tumor model have used 3D-ASL to evaluate tumor angiogenesis (29). In the present study, the TBF values of benign and intermediate tumors were lower than the TBF value of malignant tumors; this finding is consistent with the MVD results. Not only the organ injury risk but also the higher cost and poor repeatability due to intravenous injection limit the use of MR perfusion-weighted imaging with contrast agents, although CT perfusion imaging and MR perfusion-weighted imaging have achieved similar positive results $(30,31)$. Although both TBF and MVD relate to angiogenesis, how close the relationship is between them remains unknown. According to our research, TBF is significantly positively correlated with MVD. This conclusion strengthens the theory that TBF indicates angiogenesis by reflecting tissue perfusion (32). Therefore, we believe that TBF obtained from 3D-ASL is an effective biomarker of tumor angiogenesis.

According to the ROC analysis, TBF has good sensitivity and specificity in the characterization musculoskeletal tumors. We consider 3D-ASL an important sequence in discriminating between benign, intermediate and malignant musculoskeletal tumors used in combination with routine imaging methods. Additionally, TBF imaging combined with traditional structural imaging can provide direct tumor perfusion information for future operation or further treatment.

This paper has following limits: The tumors are very heterogenous. Many of these are represented by a single case. Furthermore, 3D-ASL is operator-dependent as it is subjective the choice of ROIs in the quantitative analysis. Another problem is that TBF image cannot match the structure image perfectly due to technique reasons, that will cause the deviation in measurement. Nevertheless, we believe that the inclusion of 3D-ASL in the work-up of musculoskeletal masses and the Correlation with MVD can serve as a new, fast-forward diagnostic tool for distinguishing malignant musculoskeletal tumors from their benign counterparts, the more so as 3D-ASL is a broadly available, non-invasive technique compared with contrast enhanced MRI and CT perfusion, for example.

In conclusion, the MVD represents musculoskeletal tumor angiogenesis and may reflects their biological behavior. Therefore, the MVD has clinical value in discriminating between types of tumors. Furthermore, a correlation exists between TBF and the MVD. 3D-ASL could be useful for pathological examination to a certain extent and is promising as a repeatable, completely non-invasive technique to evaluate musculoskeletal tumor angiogenesis in vivo. It has some application to determination of malignancy, but that it is premature to state that it can be used as a sole, or even dominant criterion. At least, the inclusion of 3D-ASL in established perfusion-based assessment protocols improves the evaluation of musculoskeletal masses. By the analysis of tumour perfusion, an important aspect of tumour pathophysiology-which is beyond the scope of grey-scale and conventional contrast enhanced MRI-becomes assessable. This has practical consequences for patient management: Better detection of malignant tumours by 3D-ASL and thus timely, fast-forward institution of further patient work-up, biopsy and treatment.

For the near future, a multicenter study is hoped in order to increase the number of the subject and the type of the musculoskeletal tumor, that will help the persuasion of the research.

\section{Acknowledgements}

This study was supported by the Science and Technology Program for Public Wellbeing of Ningbo municipal Bureau of Science and Technology (grant no. 2015C50022).

\section{References}

1. Campanacci M: Bone and soft tissue tumours. New York: Springer-Verlag: 199-232, 1999.

2. Weeden S, Grimer RJ, Cannon SR, Taminiau AH and Uscinska BM; European Osteosarcoma, Intergroup: The effect of local recurrence on survival in resected osteosarcoma. Eur J Cancer 37: 39-46, 2001 
3. Marulanda GA, Henderson ER, Johnson DA, Letson GD and Cheong D: Orthopaedic surgery options for the treatment of primary osteosarcoma. Cancer Control 15: 13-20, 2008.

4. Grimer RJ, Taminiau AM and Cannon SR; Surgical Subcommitte of the European Osteosarcoma Intergroup: Surgical outcomes in osteosarcoma. J Bone Joint Surg Br 84: 395-400, 2002.

5. Bradley CJ, Yabroff KR, Dahman B, Feuer EJ, Mariotto A and Brown ML: Productivity costs of cancer mortality in the United States: 2000-2020. J Natl Cancer Inst 100: 1763-1770, 2008.

6. Kransdorf MJ and Murphey MD: Radiologic evaluation of soft-tissue masses: A current perspective. AJR Am J Roentgenol 175: 575-587, 2000.

7. Siegel MJ: Magnetic resonance imaging of musculoskeletal soft tissue masses. Radiol Clin North Am 39: 701-720, 2001.

8. Wang CK, Li CW, Hsieh TJ, Chien SH, Liu GC and Tsai KB: Characteriztion of bone and soft tissue tumors with in vivo $1 \mathrm{H}-\mathrm{MR}$ spectroscopy: Initial results. Radiology 232: 599-605, 2004.

9. Johansen R, Jensen LR, Rydland J, Goa PE, Kvistad KA, Bathen TF, Axelson DE, Lundgren $\mathrm{S}$ and Gribbestad IS: Predicting survival and early clinical response to primary chemotherapy for patients with locally advanced breast cancer using DCE-MRI. J Magn Reson Imaging 29: 1300-1307, 2009.

10. Liu Q, Hu T, He L, Huang X, Tian X, Zhang H, He L, Pu W, Zhang L, Sun H, et al: Genetic targeting of sprouting angiogenesis using Apln-CreER. Nat Commun 6: 6020, 2015.

11. Fukumura D and Jain RK: Imaging angiogenesis and the microenvironment. APMIS 116: 695-715, 2008.

12. Rak JW, St Croix BD and Kerbel RS: Consequences of angiogenesis for tumor progression, metastasis and cancer therapy. Anticancer Drugs 6: 3-18, 1995.

13. Folkman $\mathrm{J}$ : What is the evidence that tumors are angiogenesis dependent? J Natl Cancer Inst 82: 4-6, 1990.

14. Weidner N, Folkma J, Pozza F, Bevilacqua P, Allred EN, Moore DH, Meli S and Gasparini G: Tumor angiogenesis: A new significant and independent prognostic indicator in early-stage breast carcinoma. J Natl Cancer Inst 84: 1875-1887, 1992.

15. Flecher CD, Unni KK and Merten F: World Health Organization classification of tumous. Pathology and genetics of tumors of soft tissue and bone. IARC Press, Lyon, pp2-10, 2002.

16. Carmeliet P and Jain RK: Angiogenesis in cancer and other diseases. Nature 407: 249-257, 2000.

17. Fukumura D, Duda DG, Munn LL and Jain RK: Tumor microvasculature and microenvironment: Novel insights through intravital imaging in pre-clinical models. Microcirculation 17: 206-225, 2010 .

18. Rak JW, St Croix BD and Kerbel RS: Consequences of angiogenesis for tumor progression, metastasis and cancer therapy. Anticancer Drugs 6: 3-18, 1995.

19. Bose S, Lesser ML, Norton L and Rosen PP: Immunophenotype of intraductal carcinoma. Arch Pathol Lab Med 120: 81-85, 1996

20. Gilles R, Zafrani B, Guinebretière JM, Meunier M, Lucidarme O, Tardivon AA, Rochard F, Vanel D, Neuenschwander S and Arriagada R: Ductal carcinoma in situ: MR imaging-histopathologic correlation. Radiology 196: 415-419, 1995.
21. Schlemmer HP, Merkle J, Grobholz R, Jaeger T, Michel MS, Werner A, Rabe J and van Kaick G: Can pre-operative contrast-enhanced dynamic MR imaging for prostate cancer predict microvessel density in prostatectomy specimens? Eur Radiol 14: 309-317, 2004

22. Marković O, Marisavljević D, Cemerikić V, Vidović A, Perunicić M, Todorović M, Elezović I and Colović M: Expression of VEGF and microvessel density in patients with multiple myeloma: Clinical and prognostic significance. Med Oncol 25: 451-457, 2008.

23. Luczynska E, Gasinska A, Blencharz P, Stelmach A Jereczek-Fossa BA and Reinfuss M: Value of perfusion CT parameters, microvessl density and VEGF expression in differentiation of benign and malignant prostate tumours. Pol J Pathol 65: 229-236, 2014.

24. Tozer GM: Measuring tumour vascular response to antivascular and antiangiogenic drugs. Br J Radiol 76: S23-S35, 2003.

25. Li Y, Yang ZG, Chen TW, Chen HJ, Sun JY and Lu YR Peripheral lung carcinoma: Correlation of angiogenesis and first-pass perfusion parameters of 64-detector row CT. Lung Cancer 61: 44-53, 2008.

26. Gordon Y, Partovi S, Müller-Eschner M, Amarteifio E, Bäuerle T, Weber MA, Kauczor HU and Rengier F: Dynamic contrast-enhanced magnetic resonance imaging: Fundamentals and application to the evaluation of the peripheral perfusion. Cardiovasc Diagn Ther 4: 147-164, 2014.

27. Loizides A, Peer S, Plaikner M, Djurdjevic T and Gruber H: Perfusion pattern of musculoskeletal masses using contrast-enhanced ultrasound: A helpful tool for characterisation? Eur Radiol 22: 1803-1811, 2012.

28. Wu WC, Wang J, Detre JA, Ratcliffe SJ and Floyd TF: Transit delay and flow quantification in muscle with continuous arterial spin labeling perfusion-MRI. J Magn Reson Imaging 28: 445-452, 2008

29. Zhang Z, Meng Q, Gao Z, Cai H, Ye Z and Wu H: Evaluation of angiogenesis of VX2 soft tissue tumor by arterial spin labeling perfusion imaging. Chin J Radiol 10: 1084-1088, 2010.

30. Bivard A, Krishnamurthy V, Stanwell P, Levi C, Spratt NJ, Davis $\mathrm{S}$ and Parsons $\mathrm{M}$ : Arterial spin labeling versus bolus-tracking perfusion in hyperacute stroke. Stroke 45: 127-133, 2014.

31. Wolf RL, Wang J, Wang S, Melhem ER, O'Rourke DM, Judy KD and Detre JA: Grading of CNS neoplasms using continuous arterial spin labeled perfusion MR imaging at 3 Tesla. J Magn Reson Imaging 22: 475-482, 2005

32. Koizumi S, Sakai N, Kawaji H, Takehara Y, Yamashita S, Sakahara H, Baba S, Hiramatsu H, Sameshima T and Namba H: Pseudo-continuous arterial spin labeling reflects vascular density and differentiates angiomatous meningiomas from non-angiomatous meningiomas. J Neuroonncol 121: 549-556, 2015. 